10.2478/gb-2020-0018

sciendo

\title{
Bleibt kreatives Schreiben ein Traum? Kreatives Schreiben als eine geistige und sprachliche Fähigkeit beim Erlernen des Deutschen als Fremdsprache in Ägypten
}

\section{Sakina Saleh MAHMOUD}

Dozent Dr.; Sohag-Universität; Ägypten;

E-Mail: sakina.saleh@art.sohag.edu.eg

„Das Schreiben ist ein accomplissement der Idee“ (Albert)

„Schreiben - Hebamme des Ichs“ (Jean Paul)

(nach: Loescher 2008: 58)

Abstract: This article is about creative writing in GFLLecture in Egypt. Writing as a skill is rarely considered in GFL- Lecture. Teachers pay attention to other skills such as reading, listening, or speaking, whereby writing is only considered receptively to promote speaking or grammar. This article is about trying to promote creative writing in GFL-Lecture and to offer new suggestions and tips. In a further step, this article deals with the presentation of some creative writing tasks that were carried out among the students of the third year at the language faculty (Al-Alsun) in Sohag. Finally, the conclusions are drawn, and results of creative writing shown.

Keywords: creative writing, association, simulation, writing with music, writingwith fantasy

\section{Einleitung}

Bemerkenswert ist, dass sich der DaF-Unterricht in den letzten Jahren in Ägypten nicht nur mit Vokabel- und Grammatikver- 
mittlung, Übersetzung der Texte ins Arabische und Lösung der Aufgaben begnügt. Es setzte sich eine kräftige Neigung zum Gebrauch kommunikativer Vorgehensweisen im DaF-Unterricht durch. Es herrscht auch eine intensive Fortbildung der DaF-Lehrer zum Gebrauch kommunikativer Unterrichtsverfahren (z. B. die DLL-Kurse und die Fortbildungskurse am GoetheInstitut). Dabei schenkt man den sich ergänzenden vier Fertigkeiten, (dem Hören, Sprechen, Lesen und Schreiben) Aufmerksamkeit insofern sie einem kommunikativen Ansatz dienen. Das Schreiben dient aber nur dazu, die gelernten Grammatikregeln und Redewendungen, die zur Kommunikation notwendig sind, anzuwenden und einzuüben. Das Schreiben vollzieht sich in Umwandlung, Ergänzung u.Ä. von „vorgefertigten Texten“, meist auf dem Rang des Satzes. Es handelt sich sozusagen um ein ,geleitetes Schreiben“. Erst auf fortgeschrittenen Niveaustufen achtet man auf freieres kommunikatives Schreiben, dem mehr Aufmerksamkeit (vgl. Solmecke 1997) geschenkt wird. In den Anfängerstufen fokussiert man sich eher auf die anderen Fertigkeiten, vor allem auf Lesen und Sprechen, obwohl das Schreiben im Allgemeinen eine ganz wichtige Rolle im Fremdsprachunterricht spielt und zur Förderung des raschen Spracherwerbs beitragen kann (Vgl. Janíková, 2005: 52).

Im vorliegenden Beitrag beschränke ich mich auf eine der vielen verschiedenen Schreibmethoden, nämlich auf das kreative Schreiben. Aus meiner Erfahrung mit den Studenten in Ägypten ist mir klar geworden, dass es DaF-Lernenden schwer fällt, ihre Gedanken über ein bestimmtes Thema schriftlich frei auszudrücken. Einerseits, weil die meisten DaF-Lehrwerke über nur ganz geringe Aufgaben verfügen, die das kreative Schreiben bei den Lernenden fördern (vgl. z. B. die Lehrwerke, Tangram aktuell, Lagune, Aussichten, Linie 1 u.a.) und andererseits, weil das Schreiben im kommunikativ orientierten DaF-Unterricht nicht im Vordergrund steht (vgl. Krumm 1993: 23). Ein kommunikativer Unterricht legt vor allem großen Wert 
auf die gesprochene Sprache, wobei das Schreiben nur gebraucht wird, um das Sprechen zu fördern.

Das Schreiben spielt aber eine wesentliche Rolle beim Erlernen und Beherrschen einer Fremdsprache. Mithilfe des Schreibens kann man geistige und sprachliche Fähigkeiten fixieren. „Für den Fremdsprachenunterricht ist Schreiben eine wichtige Kontaktmöglichkeit zu einer fremden Struktur" (Heyd 1997: 181). Am Ende des Studiums sollten Studenten auch fähig sein, sich in der Fremdsprache z.B. bei einer Firma zu bewerben; darzustellen, warum gerade diese Firma interessant ist und warum man selbst für den Posten an dieser Firma geeignet ist. Studenten, die weiter in der Wissenschaft tätig sein wollen, sollten ihre eigenen Gedanken und Meinungen schriftlich flüssig darstellen können. Und ganz allgemein: Häufig geschieht der erste Kontakt mit einer fremden Institution schriftlich.

Aus diesen Gründen sollte das kreative Schreiben im DaFUnterricht in Ägypten als Mittel zum besseren Spracherwerb genutzt werden. Deswegen geht es in diesem Beitrag nicht um eine Fehleranalyse der schriftlichen Produktion der Lernenden oder um einen ausführlichen Vergleich der Schreibaufgaben in den unterschiedlichen DaF-Lehrbüchern1, sondern um die Förderung des kreativen Schreibens im DaF-Unterricht durch einige Aufgaben und Schreibspiele.

Der Beitrag umfasst zwei Teile. Im ersten Teil geht es um den Begriff kreatives Schreiben. Es wird auch gezeigt, welche Bedeutung das kreative Schreiben für den Erwerb des Deutschen als Fremdsprache hat. Im Weiteren werden auch einige Unterrichtsmethoden erläutert.

${ }^{1}$ Es ist hier zu erwähnen, dass es einige Ansätze im Rahmen der Germanistik in Ägypten gibt, die sich auch mit der Fertigkeit ,schreiben“ beschäftigen, aber andere Schwerpunkte und andere Ziele haben (vgl. u.a. Abdalla (2010), Yehia (2015), Mostafa (2017), Kook 2018)Demgegenüber wird im vorliegenden Beitrag das kreative Schreiben als ein Mittel zur Förderung des Spracherwerbs bei den Lernenden in Ägypten geschildert. 
Im zweiten Teil werden zwei Schreibaufgaben dargestellt, die von den Studenten des 3. Studienjahrs der Sprachenfakultät (Al-Alsun) in Sohag durchgeführt wurden. Es wird auch gezeigt, welche Folgen die Einführung des kreativen Schreibens auf das Lernverhalten der Studenten hat.

\section{Was ist gezieltes kreatives Schreiben?}

Obwohl die Sprachdidaktik des Faches Deutsch als Fremdsprache ab Mitte der 1980er Jahre der Fertigkeit des Schreibens größere Aufmerksamkeit geschenkt hat (vgl. Fischer-Kania 2008:481), hat sich diese Einsicht für den DaF-Unterricht in Ägypten bislang nicht ganz durchgesetzt. „Freie“ Schreibfertigkeiten werden erst in den fortgeschrittenen Stufen ein wenig gefördert, da der Schreibprozess als hochkomplex angesehen wird. Darüber hinaus muss man feststellen, dass es den Studenten (auch den fortgeschrittenen Studenten) schwer fällt, „frei“"zu schreiben. Schreibübungen werden meistens als Hausaufgaben gegeben und sie werden als langweilig und schwierig empfunden. Die Frage ist, wie könnte man die Studenten - und auch die Lehrer - dazu motivieren, mehr zu schreiben, Wie kann man Lernende und manche Lehrer davon überzeugen, dass das Schreiben mehr Funktionen hat, als sie glauben? Und dass die Fremdsprache auch durch Schreiben schnell und gut erlernt werden kann (vgl. Benešová, 2005, S.27-28).

Schreiben hat viele Formen, die den unterschiedlichen Zwecken dienen können. Man unterscheidet die folgenden Typen vom Schreiben:

- das personale Schreiben: Bei diesem Schreibtyp gibt man Auskunft über sich selbst, z.B. etwas im Tagebuch eintragen, Notizen schreiben, einen Erlebnisbericht verfassen, eine Urlaubspostkarte schreiben usw.),

- das pragmatische Schreiben im Alltag: Zu diesem Typ des Schreibens zählen z. B. ein Formular ausfüllen, eine E-Mail 
verfassen, Kochrezepte zusammenstellen, Glückwünsche schreiben, eine Einladung formulieren, eine Orts- bzw. Wegbeschreibung für eine Einladung eingeben/ eine Danksagung schreiben etc.),

- das Verfassen expositorischer Texte: Hier kann man z. B. Notizen zu einem Vortrag machen, eine Stellungnahme schriftlich eingeben, einen Beitrag für eine Fachzeitschrift schreiben),

- das kreative Schreiben: Dieses Schreibverhalten ermöglicht z. B. das Verfassen von kleinen Gedichten, Erzählungen, Cartoontexten, Hörspielen und Theaterstücken sowie Liedern und Parodien) (vgl. Kast 1999).

Da sich vorliegender Beitrag an kreatives Schreiben richtet, wird hier auf die Definition des kreativen Schreibens eingegangen. Der Begriff des kreativen Schreibens stammt aus dem Englischen creative writing, das in den letzten Jahren des 19. Jahrhunderts als Fach in den Curricula der amerikanischen Universitäten erscheint.

Damals wurde es zur Bezeichnung von Seminaren gebraucht, in denen die Studenten der Literaturwissenschaft ihre experimentellen Schreiberfahrungen sammeln sollten (Glindemann 2000: 1 ff.). Das Ziel war, Schreibtechniken zu erwerben, damit man sich selbst in richtig gestaltender Sprache ausdrückt, vor allem für die beruflichen Situationen und für die Erweiterung vom produktiven Denken (vgl. Fritzsche 1994: 159 ff.). Im Laufe der Zeit wurde creative writing zu einem Schreibverhalten, mit dem der Schreiber sich als Person ausdrücken konnte und sich phantasieren lässt. Kreatives Schreiben ist also „das Abfassen freier, persönlich verantworteter Texte.“ (Merkelbach 1993: 137).

Im Deutschen bezeichnet das kreative Schreiben auch Schreibansätze, die von der Hypothese ausgehen, dass der Prozess des Schreibens hauptsächlich ein kreativ-sprachlicher 
Prozess ist, auf den die Schreibenden methodisch gerichtet werden können. Kreatives Schreiben unterscheidet sich damit vom klassischen Schreibunterricht dadurch, dass man beim kreativen Schreiben den Schwerpunkt vor allem auf den Prozess des Schreibens selbst legt und diesen Prozess "durch assoziative, gestaltende und überarbeitende Methoden trainiert" (vgl. Böttcher 1999: 7). Eine einheitliche Beschreibung des Inhalts vom kreativen Schreiben gibt es kaum, weil die vorhandenen Ansätze zu diesem Schreibverhalten sich in den Methoden, Verfahren, Grundlagen und Zielen stark differenzieren (vgl. von Werder 2004: 21ff.).

Kreatives Schreiben ist also die Kunst, frei zu verfassen, zu assoziieren und individuelle Anforderungen zum Ausdruck zu bringen. Der Schritt zum kreativen Schreiben beginnt da, wo die Lernenden die "graue Zone" der reinen Widergabe und Einübung des Erlernten überschreiten und sich etwas Neues zutrauen. Es geht also beim kreativen Schreiben um die Fähigkeit zur Überwindung der Barrieren und Blockaden beim Schreiben und zum kreativen Einsatz der Sprache. Auf den Fremdsprachenunterricht bezogen, bedeutet das, dass man versucht auch dort, einen freien Gedankenaustausch zu fördern. „Im weiteren Sinn ist das Kreativ-sein vielmehr gleichbedeutend mit Entdecken, Basteln, Ausprobieren und Spielen, weil aus diesen Tätigkeiten meistens etwas Neues, Originelles hervorgeht" (Lorey 2000: 9).

Die Erfahrungen mit dem kreativen Schreiben, die ich mit meinen Studenten gemacht habe und die ich in Abschnitt 2 zeigen werde, bestätigen noch die Auffassung von Kast. Er hält fest, dass das freie Schreiben und die Kreativität zusammen passen (Kast 1999: 127). Anstatt, dass man sich allein auf Orthographie, Diktate und Aufsätze im DaF-Unterricht konzentriert, soll auch auf den kreativen Umgang mit der Sprache eingegangen werden. Dies soll einen positiven Einfluss auf Ausdrucksund Wortschatzvermögen von den Lernenden haben. 
Das kreative Schreiben gilt also nicht als eine einzelne Sonderveranstaltung, sondern als ein Unterrichtsverfahren, das zum Spracherwerb auf allen Ebenen und in allen Kontexten des Erwerbs beiträgt und gleichzeitig fächerübergreifend gebraucht werden kann. Durch den Einsatzvon authentischen und persönlichen Faktoren im Schreibunterricht sowie auch durch die lerner- und situationsorientierte Unterrichtsform haben Lernende die Möglichkeit, ihre individuellen Persönlichkeiten in den Unterricht einzubeziehen. Das Schreiben ist durchaus nicht mehr als reines Abstrahieren von verbalen Formen zu betrachten, sondern erschließt sich den Lernenden gleichzeitig als Medium zur Lebensbewältigung. Dieses Bedenken vereinfacht den Einstieg in die Textproduktion, regt die Lust am Schreiben an und erweckt die Lust am Erzählen gleichermaßen wieder. Die große Motivation der Lernenden führt deutlich zu einem schnellen und fundierten Schriftspracherwerb. (vgl. Brock 2003: 129)

Vor allem im DaF-Unterricht können Schreibspiele oft als Einstiegsübungen in komplexere Methoden vom kreativen Schreiben gebraucht werden. Sie tragen dazu bei, einen lustvollen Weg zum sprachlichen Ausdruck zu finden. Dazu stehen unterschiedliche Methoden zur Verfügung. Im Vordergrund des Verfahrens zum kreativen Schreiben steht der Spaß am Umgang mit der Sprache. Durch den Einsatz von kreativen Schreibmethoden können Geschichten entstehen und Texte verfeinert werden.

\section{Methoden des kreativen Schreibens}

Kreatives Schreibverhalten führt nicht nur zur Förderung der Desautomatisierung beim Schreiben, sondern lässt auch Denkund Wahrnehmungsstereotypen durchbrechen. Mit Hilfe von Schreibaufgaben werden Lernende motiviert. Die Vertrautheit des kreativen Schreibens führt zur Verbesserung des sozialen 
Klimas innerhalb einer Lerngruppe und ermöglicht das Selbstund Fremdverstehen. Es führt auch zu weiteren ästhetischen Kompetenzen, u.a. Selbstgestaltung, Selbstausdruck und Problemlösen. Das kreative Schreiben verfügt über spezifische Methoden. Die Methoden des kreativen Schreibens können zur Motivierung der Lernenden gebraucht werden. Dabei können die verschiedensten Wege der Inspiration für die Textproduktion eingesetzt werden. Kreatives Schreiben umfasst eine Vielzahl von kreativen Methoden, wie z. B. Brainwriting, Cluster, Freewriting, Journal-Writing oder auch Brainstorming und das Mind-Map. Nicht alle wurden jedoch ausschließlich für den Schreibunterricht entwickelt, wie man es aus den erwähnten Methoden erschließen kann.

Nach Böttcher (1999) kann man die sechs folgenden Methodengruppen vom kreativen Schreiben unterscheiden:

1. Das assoziative Schreibverfahren: Diese Methode teilt man in zwei Untergruppen, nämlich eine spielerisch experimentelle, z. B. Cluster, Gegensatzcluster, automatisches Schreiben, Schreiben zu Reizwörtern, Wortbörse, Wörterfinden, bildliche Assoziationen, sternförmig Denken; meditativ: Fantasiereise, Metaphern-Meditation, Wahrnehmungsübungen usw.;

2. Schreibspiele: Dazu gehört Folgendes: Geschichten reihum verfassen, Geschichten erwürfeln, Gedichte reihum entwickeln, Wörter finden, z.B. Wörtersack, Wörterkiste/ Wörterkoffer, Klopfwörter, Landschaftsmalerei, SchatzRegal, usw.;

3. Schreiben nach Mustern, Regeln und Vorgaben: Diese Gruppe umfasst inhaltliche Vorgaben, formale Kriterien, strukturelle Regeln, literarische und textorientierte Muster, Gedichte aller Arten und mit allen Sinnen, Zeilenumbrechen, Schneeballgedicht, Rondell, Elfchen, Akrostichon, das serielle Schreiben, Schreiben von mathematischen Vorgaben, Geschichten zu Wörtern, Kurzromane, 
das metaphorische Schreiben, assoziative Texte verfeinern, Texte reduzieren und zusammenfassen;

4. Schreiben von literarischen Texten: $\mathrm{Zu}$ dieser Methode gehören z. B. Zeilen füllen, Lückentexte, Texte reduzieren, eine Geschichte bzw. einen Text zu Ende schreiben, Perspektivenwechsel bzw. Werbetexte verfassen;

5. Schreiben zu Stimuli: Hier findet man Schreiben mit Musik, Bildern, Tanzen, Gegenständen, Landschaft, Ort, Fantasiereisen, sowie Schreiben mit mathematischen Begriffen, Textzeilen, Wörternusw.;

6. Weiterschreiben an kreativen Texten: Zu dieser Methode gehören vor allem kreative Bearbeitungsverfahren, kriterienorientierte Schreibverfahren, sukzessives Ergänzen von Textanfängen, Textreproduktion, Zeilen umbrechen, Textteile, Rondell, Textlupe, Operieren, über den Rand schreiben, Spezialisten, Weiterarbeiten in Stationen (Böttcher; 1999: 24 ff.)

Die oben genannten Methoden werden zwar sechs verschiedenen Gruppen zugeordnet, können aber nicht getrennt voneinander betrachtet werden, da sie viele Überschneidungen aufweisen und sich in verschiedenen Schreibaufgaben miteinander verbinden lassen. Sie können nämlich auf jede Lernstufe eingesetzt werden.

Im folgenden Abschnitt werden zwei Aufgaben zur Förderung des kreativen Schreibens im DaF-Unterricht an der Germanistikabteilung der Sohag-Universität vorgeführt, und es wird gezeigt, dass die Förderung des kreativen Schreibens bei den Studenten einen positiven Effekt auf Ihr Lernverhalten, Lernfreude und Lernresultate hatte.

\subsection{Kreatives Schreiben an der Germanistikabteilung in Sohag}

Die Fragestellung der durchgeführten Untersuchung bildet die Hypothese, dass das kreative Schreiben den Spracherwerb 
unterstützt und die Interaktion im DaF-Unterricht fördert. Die vorliegende Untersuchung soll aufzeigen, dass das freie kreative Schreiben dazu dienen kann, dass man im DaF-Unterricht sowohl den Lernerfolg als auch die Freude am Lernen zusammenbringen kann. Dies zeigteine Dokumentation verschiedener Schreibaufgabenim DaF-Unterricht, die in Schreibgruppen mit Studenten des 3. Studienjahrs an der Germanistikabteilung in Sohag durchgeführt wurde. Die Studenten lernen Deutsch als Fremdsprache und arbeiten gern in Gruppen- oder Partnerarbeit. Die Wahl der Gruppenmitglieder wurde durchgeführt, indem stärkere und schwächere Teilnehmer miteinander kombiniert wurden, sodass alle Gruppen fast das gleiche Sprachniveau hatten. Es haben daran 43 Studenten teilgenommen, die in 8 Gruppen mit je 5/6 Personen eingeteilt wurden. Die Arbeitsatmosphäre in den Lerngruppen war äußerst angenehm.Die Beteiligungder Studenten an den kreativen Schreibaufgaben war erfreulich und gestrebt. Die Lerngruppen waren sehr begeistertund motiviert (Im Gegensatz zu ihrem früheren Verhalten zu denerforderlichen Schreibaufgaben). Im Folgenden werden zwei Beispiele aus der Dokumentation der Schreibaufgaben von den Studenten dargestellt:

Das eine Aufgabenbeispiel zum kreativen Schreiben, das den Probanden vorgelegt wurde, heißt: Zehn Wörter... ein Text ${ }^{2}$. Dabei wurde den Studenten folgender Kasten mit zehn Wörtern vorgelegt:

Wohnung - Schiff - Arzt - Tasse - Termin - Sonnenschirm - Handy - Baum - Fahrrad - Pullover

Das andere Beispiel ist Folgendes:

Schauen Sie sich die zwei Bilder genau an, wählen Sie eins aus und schreiben Sie dazu eine Geschichte:

2 Bei dieser Aufgabe kann die Lehrperson die angegebenen Wörter nach dem gewünschten Thema bearbeiten bzw.erweitern, so dass die Lernenden es üben und wiederholen können. 

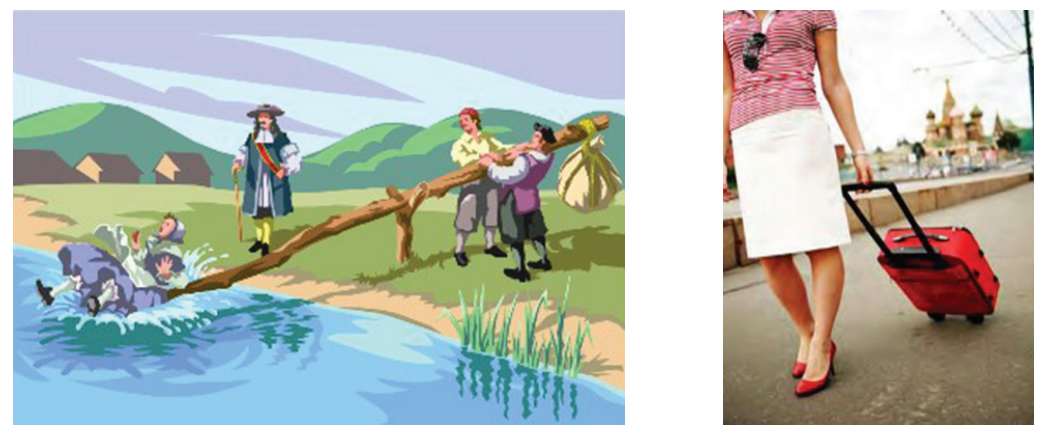

Der Grund für die Auswahl dieser zwei Methoden ist, dass ich diese Methoden mehrmals mit meinen Studenten ausprobierte und jedes Mal beobachtete, dass sie mit diesen Methoden gute Ergebnisse erzielten und sehr zufrieden und motiviert waren.

Die Schritte der angeführten Aufgaben sind folgendermaßen abgelaufen:

Schritt 1: Als Einführung zum Thema wird die Aufgabe (ein Blatt Papier mit den 10 Wörtern/Bilder) angegeben. Die Lernenden bildeten Hypothesen, wie sie ihre Geschichte wohl einleiten sollten.

Schritt 2: Die Lernenden arbeiten in Gruppen. Sie tauschen ihre Gedanken und Ideen aus, dabei fertigen einige ein Cluster oder Mindmap an. Wörterbücher dürfen sie auch gebrauchen.

Schritt 3: Nach dem Formulieren der einzelnen Geschichten werden sie mit Hilfe der Lehrkraft überarbeitet, da es manchen Lernenden schwerfällt, ihre eigenen Texte alleine zu revidieren.

Schritt 4: Lernende präsentieren ihre Geschichten. Andere hören ihnen zu. Danach gab es Diskussionen. Sie tauschen ihre Meinungen gegenseitig aus. Schließlich werden die Geschichten an die Pinnwand gehängt.

Die Ergebnisse der Schreibspiele sind meistens sehr positiv. Mit Hilfe von kreativen Schreibspielen entstehen sehr gute und fantasievolle Texte, die Lernenden erzielen sehr guten Erfolg, wobei die Zusammenarbeit in den Gruppen gefördert wird. 
Dank dieser und weiterer ähnlicher Schreibspiele wird das Schreiben eine von den Lieblingsaktivitäten der Lernenden.

Positiv an diesen Aufgaben ist, dass man sie ganz spontan und ohne große Vorbereitung im Unterricht einsetzen kann. Sie erfordern keine ausführlichen Erklärungen oder großen Zeitaufwand beim Vorbereiten der Materialien.

Das Gefühl, dass man anhand einer Anzahl von Wörtern oder eines Bildes Geschichten erfindet, Handlungen entwickelt, Ideen schriftlich formuliert und zu Handlungsträger eines kurzen Textes bzw. einer kleinen Geschichte wird, hat durchaus einen besonderen Eindruck bei den Lernenden. Das alles ist dem kreativen Schreiben zu verdanken.

\subsection{Folgen des kreativen Schreibens}

Die Übungen zum Kreativen Schreiben fußen auf Zusammen arbeit und Kreativität, die Lernenden versuchen, Lösungen für Probleme in der Gruppe zu finden, Texte in Kooperation zu erstellen und eine gemeinsame Bewertung der Texte vorzunehmen. Auf der einen Seite trägt dieses Schreibverhalten zur Förderung des Teamgeistes unter den Lernenden bei, auf der anderen Seite hilft es den Lernenden, die Konkurrenz in den Gruppen durch Kooperation zu ersetzen.

Dank des kreativen Schreibens können Lernende mit Spaß schreiben, eigene Texte verfassen und in die deutsche Sprache -als Fremdsprache- ihre schriftlichen Experimente machen. Sie warten neugierig auf die Texte ihrer Kollegen und genießen das eigene Probieren und Trainieren ihrer Fantasie. Darüber hinaus ist Schreiben in der Gruppe spannend und durchbricht Schreibblockaden. Lernende reagieren immer begeistert, wenn ihnen die Gelegenheit zum kreativen Schreiben geboten wird. Es zeigte sich, dass kreatives Schreiben den DaF-Unterricht und den Erwerb des Deutschen als Fremdsprache bereichert. Dadurch, dass die Lernenden zum Ausdrücken ihrer eigenen Gedanken neue Wörter brauchten und Clusters und Mindmaps anfertigten, 
konnten sie ihren Wortschatz auch schnell ausbauen. Der Versuch, kompliziertere Satzgefüge zu formulieren, führte auch zu einem größeren Interesse an der Syntax der Fremdsprache. Die Erweiterung des Vokabulars und der Syntax können dann von der Lehrperson als Ausgangspunkt für einen gezielten Unterricht genommen werden, der besser von den Lernenden aufgenommen und memoriert wird, da er durch ihre eigenen Bedürfnisse gesteuert wird. Daher wäre es nützlich und empfehlenswert, das kreative Schreiben in die Curricula der ägyptischen Germanistikabteilungen miteinzubeziehen und diesem Schreibverhalten eigenständigen Unterricht, neben weiteren Fächern wie Übersetzung, Literatur- und Grammatikunterricht u.a. zu widmen.

\subsection{Corona-Pandemie als Auslöser für die Förderung des kreativen Schreibens an den ägyptischen Schulen und Universitäten.}

Wegen der bestehenden Umstände der Corona-Pandemie beschließen ägyptische Ministerien für Bildung und Hochschulbildung, dass die Abschlussbewertung der ägyptischen Schüler und Studenten für das 2. Semester des Schul- und Studienjahres 2020 durch Verfassen eines eigenständigen wissenschaftlichen Beitrags zu den verschiedenen Fächern durchgeführt wird. Die Studenten werden gebeten, an Stelle der gewöhnlichen Abschlussprüfungen, ein Thema frei auszuwählen und es schriftlich zu behandeln.

Dieses Verfahren fördert die schriftliche und geistige Kreativität der Schüler und der Studenten und regt das Denken an. Viele fanden die Idee gut, da sie mit dieser Vorgehensweise mit Spaß lernen konnten und nicht einfach viel Material für die üblichen schulischen und universitären Prüfungen auswendig lernen müssen. Zukünftig wünscht man sich einen richtigen Einbezug des kreativen Schreibens in den ägyptischen Schulen und Universitäten, der den Lernenden viel Platz zum Denken, 
Assoziieren und zur Kreativität bietet. Es ist wünschenswert, das kreative Schreiben zu einem Hauptmotiv nicht nur beim Erlernen von Fremdsprachen, sondern auch beim schulischen und universitären Lernen zu betrachten.

\section{Schlussfolgerungen}

Wie man sieht, gibt es also viele Gründe, dem kreativen Schreiben die Chance zu geben, Teil der ägyptischen Curricula in den Schulen und den Universitäten zu werden. Die Lernenden haben dadurch die Möglichkeit mit der Muttersprache oder der Fremdsprache $\mathrm{zu}$ experimentieren. Das kreative Schreiben nimmt den persönlichen Bezug zu den Texten als Ausgangspunk wahr und dadurch entsteht die Motivation. Kreatives Schreiben lässt die Schreiber die Freude über den produzierten Text erleben, fördert die Zusammenarbeit in der Gruppe und motiviert die Schreibenden, nicht ratlos mit einem leeren Blatt Papier alleine zu sein. Das Einteilen der talentierten oder weniger talentierten Studenten in unterschiedliche Gruppen und die Zusammenarbeit baut die psychischen Barrieren und den unerwünschten Stress ab. Daher wünscht man sich zukünftig eine positivere Haltung zur Einführung des kreativen Schreibens in den ägyptischen Schulen und Universitäten.

\section{Literatur}

Abraham, Ulf(2014): "Kreatives” und "poetisches” Schreiben. In: Helmuth Feilke, Thorsten Pohl (Hrsg.): Schriftlicher Sprachgebrauch - Texte verfassen (Deutschunterricht in Theorie und Praxis Band 4). S. 364-381.

Barbara Glindemann (2000): Creative writing. Zu den kulturellen Hintergründen und zum literaturwissenschaftlichen und institutionellen Kontext im Vergleich zwischen England, USA und Deutschland. Dissertation. Hamburg. 
Benešová, Pavla (2005): Brünner Hefte für Germanistik. 1. Brno: Masarykova univerzita. Merkelbach, Valentin (1993):

Kreatives Schreiben. 1. Braunschweig: Westermann.

Brenner, Gerd (1990): Kreatives Schreiben: Ein Leitfaden Für die Praxis. 1,. Frankfurt am Main.

Brock, Ursula (2003): Kreatives Schreiben im vielsprachigen Deutschunterricht, Inaugural-Dissertation in der Philosophischen Fakultät II (Didaktik des Deutschen als Zweitsprache, Linguistik, Soziologie) der Friedrich-AlexanderUniversität Erlangen Nürnberg.

Böttcher, Ingrid (Hrsg.) (1999): Kreatives Schreiben. Grundlagen und Methoden. Beispiele für Fächer und Projekte. Frankfurt am Main: Cornelsen Scriptor.

Dauvillier, Christa; Lévy-Hillreich, Dorothea (2004): Spiele im Deutschunterricht. München: Goethe-Institut.

Fišer, Zbyněk (2001): Tvưrčí psaní. Brno. Paido.

Fritsche, Joachim (1994): Zur Didaktik und Methodik des

Deutschunterrichts 2. Schriftliches Arbeiten. Stuttgart: Klett.

Heyd, G. (1997): Aufbauwissen für den Fremdsprachenunterricht (DaF). Ein Arbeitsbuch. Kognition und Konstruktion. Tübingen: Narr.

Hinrichs, Beatrix (2011): Kreatives Schreiben - ein Weg zur Förderung der Schreibkompetenz von Schülern mit Deutsch als Zweitsprache im Deutschunterricht. In: Deutsch als Fremdsprache in allen Fächern.

Janíková, Věra (2005): Didaktik des Deutschen als Fremdsprache: Anemrkungen zu aktuellen Themen mit Aufaben zum reflektierten Selbststudium. 1. Brno : Masarykova univerzita.

Kast, Bernd (1999): Fertigkeit Schreiben. Fernstudieneinheit 12. Berlin: Langenscheidt.

Krumm H.-J (1993): Kulturgeprägte Einflüsse auf das Schreiben und ihre möglichen Einflüssen für den Schreibunterricht Deutsch als Fremdsprache. Tütken, G., Neuf-Münkel, G. (Hrsg.). Schreiben im DaF-Unterricht an Hochschulen 
und Studienkollegs. Bd. 1: Forschungsergebnisse - Didaktische Konzeption - Übungsformen. Regensburg: Fachverband Deutsch als Fremdsprache, S. 23-33

Loescher, Jens (2008): Schreiben, Denken, Sprechen: in der Fremdsprache. Ein Neuansatz.

Lorey, Christoph (2000): Kreatives Schreiben im ersten Jahr Deutsch als Fremdsprache? Dreizehn Fragen und Antworten mit anregenden Aktivitäten.

Merkelbach, Valentin (1993): Kreatives Schreiben. 1. Braunschweig: Westermann Schulbuchverlag.

Osama Taha Kook, Amina Al-Shaimaa (2018): Zur Förderung der Schreibfertigkeit durch den Einsatz von „Lernen an Stationen“ im DaFUnterricht. Empirische Untersuchung an der GUC Masterarbeit im Fachgebiet Methodik- Didaktik im Fachbereich Deutsch als Fremdsprache. Kairo. Pommerin, Gabriele (1996): Kreatives Schreiben. Weinheim und Basel.

Porsch, Raphaela (2010): Schreibkompetenzvermittlung im Englischunterricht - Eine bundesweite Umfrage. In: Zeitschrift für Fremdsprachenforschung, 21: 1, S. 53-77

Truffer, Josefine (2007): Kreatives Schreiben in der Primarschule. Diplomarbeit an der Pädagogischen Hochschule Wallis.

Von Werder, Lutz (2004): Lehrbuch des kreativen Schreibens. 4. Auflage. Berlin.

\section{Internetquellen}

www.teachsam.de/deutsch/d_ubausteine/schr_ub/eroert_ub/ ero_them/tou/ero_them_tou1_2.htm. 